\title{
Asteroid encounters suitable for mass determinations
}

\author{
A. Galád ${ }^{1}$ and B. Gray ${ }^{2}$ \\ 1 Astronomical Institute, Faculty of Mathematics, Physics and Informatics, Comenius University, Bratislava, Slovak Republic \\ 2 Project Pluto, 168 Ridge Road, Bowdoinham, ME 04008, USA
}

Received 19 December 2001 / Accepted 28 May 2002

\begin{abstract}
As in a previous paper (Galád 2001), the search for effective perturbers among asteroids is done using the same method and during the same time period. The only difference is in the number of asteroids that were processed -24599 instead of 9511. Special attention is paid to comparison between perturbations due to (2) Pallas and (10) Hygiea. It is confirmed that the latter has a larger effect on the motion of main belt asteroids, perhaps by a factor of three. This is a reason to include its mass in asteroid orbit determinations.

In addition to the Big Four main belt asteroids - (1) Ceres, (2) Pallas, (4) Vesta, (10) Hygiea - the masses of many other large asteroids, such as (11) Parthenope, (13) Egeria, (15) Eunomia, (16) Psyche, (24) Themis, (29) Amphitrite, (39) Laetitia, (45) Eugenia, (52) Europa, (65) Cybele, (121) Hermione, (451) Patientia, and (511) Davida, could be achieved by the end of this decade using astrometric data. In general, over the next decade small asteroids (with much higher numbers than above) will be used more thoroughly for mass determination of large asteroids.
\end{abstract}

Key words. minor planets, asteroids - astrometry - ephemerides

\section{Introduction}

The number of papers devoted to mass determinations of large asteroids is growing in recent years. There are several opportunities that enable such a determination - the discovery of satellites of asteroids (e.g. Merline et al. 1999; Merline et al. 2000; Merline et al. 2001; Brown \& Margot 2001; Storrs et al. 2001), measurements by space probes that visited asteroids (e.g. Thomas et al. 1999). Also important are the growing number of astrometrical measurements and their increased precisions (see e.g. Carpino \& Knežević 1996; Hilton et al. 1996; Viateau \& Rapaport 2001; Michałak 2001 and references therein).

It was realized that after a small asteroid had passed close enough to a large one, the perturbing effects of the latter (on the motion of the former) could be significant. Large asteroid mass should therefore not be neglected - it can be determined through the study of the motion of the affected small asteroid. The three largest main belt asteroids - (1) Ceres, (2) Pallas, and (4) Vesta - contain about half the mass of the belt. Thus, it is believed their masses are sufficient enough to be included (along with planetary masses) for high precision orbit computations of the other asteroids in the inner part of the Solar System. Their masses have been accurately determined by this astrometric method.

However, it would be beneficial to determine masses of the other large main belt asteroids similarly. Not only is this

Send offprint requests to: A. Galád, e-mail: galad@fmph.uniba.sk useful for physically characterizing asteroids; it is helpful for precise long-term orbit determinations of several smaller objects that come close enough to large asteroids to be measurably affected. The discovery rate of smaller asteroids is rapidly growing, and these may greatly aid in future mass determinations, as their deviations from the ephemeris positions are evident several years (or even decades) before and after an encounter with larger objects.

The aim of this paper is to renew the previous list by Galád (2001) (hereafter referred as Paper I) of close encounters suitable for asteroid mass determination by astrometric methods. It reflects the role of the increased number of known asteroids and the larger amount of astrometric data. Included are only the most important encounters. In Paper I, a database of the first 9511 numbered asteroids were used. Here, the first 24599 numbered asteroids are discussed, excluding 29 Centaurs and transneptunian objects. Asteroid masses are not computed here.

\section{Orbital elements}

The initial osculating orbital elements for epoch April 1, 2001 (JD 2452 100.5), were taken from Bowell et al. (1994). Perturbations due to all planets, Moon, and the three largest main belt asteroids - (1) Ceres, (2) Pallas, (4) Vesta - were taken into account.

We derived orbital elements for several other epochs to the past and the future from the initial elements. However, perturbations due to asteroids were not included. Adjacent 
Table 1. Asteroids heavily perturbed by (1) Ceres $(P \geq 60 \mathrm{~km} \mathrm{~s}, D=913 \mathrm{~km})$, which were well observed before and after an encounter. The dates of the first astrometric measurements of the perturbed asteroid available in MPC data are given in the last column.

\begin{tabular}{rlcccrc}
\hline \hline number & name & $\begin{array}{c}\text { date } \\
\text { year } / \mathrm{m} / \mathrm{d}\end{array}$ & $\begin{array}{c}r \\
{[\mathrm{AU}]}\end{array}$ & $\begin{array}{c}v \\
{\left[\mathrm{~km} \mathrm{~s}^{-1}\right]}\end{array}$ & $\begin{array}{c}P \\
{[\mathrm{~km} \mathrm{~s}]}\end{array}$ & $\begin{array}{c}\text { obs } \\
\text { year }\end{array}$ \\
\hline $\mathbf{( 2 5 7 2 )}$ & Annschnell & $1971 / 3 / 26$ & 0.012011 & 4.786 & 88.5 & 1950 \\
$\mathbf{( 4 5 4 )}$ & Mathesis & $1971 / 11 / 23$ & 0.021613 & 2.928 & 80.4 & 1900 \\
$\mathbf{( 3 6 4 3 )}$ & Tienchanglin & $1972 / 9 / 11$ & 0.008282 & 2.765 & 222.2 & 1937 \\
$\mathbf{( 1 1 ~ 1 3 7 )}$ & 1996 XE19 & $1975 / 11 / 4$ & 0.007778 & 6.615 & 98.9 & 1949 \\
$\mathbf{( 5 5 6 4 )}$ & 1991 VH2 & $1975 / 12 / 21$ & 0.030697 & 1.446 & 114.6 & 1951 \\
$\mathbf{( 5 3 4 )}$ & Nassovia & $1975 / 12 / 24$ & 0.022585 & 2.752 & 81.8 & 1904 \\
$\mathbf{( 3 3 4 4 )}$ & Modena & $1980 / 9 / 27$ & 0.021136 & 2.386 & 100.9 & 1955 \\
$\mathbf{( 7 2 9 8 )}$ & 1992 WM5 & $1982 / 5 / 23$ & 0.043757 & 1.394 & 83.4 & 1953 \\
$\mathbf{( 7 8 6 )}$ & Bredichina & $1983 / 4 / 10$ & 0.028102 & 2.598 & 69.7 & 1914 \\
$\mathbf{( 6 3 2 5 )}$ & 1991 EA1 & $1983 / 9 / 18$ & 0.015592 & 3.603 & 90.6 & 1955 \\
$\mathbf{( 3 4 8 )}$ & May & $1984 / 9 / 2$ & 0.042422 & 0.794 & 151.0 & 1892 \\
$\mathbf{( 5 3 0 3 )}$ & Parijskij & $1996 / 9 / 11$ & 0.005537 & 2.736 & 335.8 & 1971 \\
\hline
\end{tabular}

Table 2. Asteroids heavily perturbed by (4) Vesta $(P \geq 20 \mathrm{~km} \mathrm{~s}, D=501 \mathrm{~km})$ which were well observed before and after an encounter.

\begin{tabular}{rlcccrc}
\hline \hline number & name & $\begin{array}{c}\text { date } \\
\text { year/m/d }\end{array}$ & $\begin{array}{c}r \\
{[\mathrm{AU}]}\end{array}$ & $\begin{array}{c}v \\
{\left[\mathrm{~km} \mathrm{~s}^{-1}\right]}\end{array}$ & $\begin{array}{c}P \\
{[\mathrm{~km} \mathrm{~s}]}\end{array}$ & $\begin{array}{c}\text { obs } \\
\text { year }\end{array}$ \\
\hline $\mathbf{( 5 2 0 5 )}$ & $\mathbf{1 9 8 8 ~ C U 7}$ & $1977 / 5 / 12$ & 0.002858 & 3.731 & 78.8 & 1954 \\
$\mathbf{( 2 1 ~ 2 2 5 )}$ & $\mathbf{1 9 9 5}$ GQ1 & $1981 / 1 / 12$ & 0.014154 & 1.651 & 36.0 & 1972 \\
$\mathbf{( 3 0 5 7 )}$ & Malaren & $1983 / 1 / 19$ & 0.024393 & 1.646 & 20.9 & 1952 \\
$\mathbf{( 8 3 3 1 )}$ & Dawkins & $1988 / 1 / 19$ & 0.007964 & 1.475 & 71.6 & 1982 \\
$(\mathbf{1 7})$ & Thetis & $1996 / 6 / 16$ & 0.019377 & 1.168 & 37.1 & 1852 \\
\hline
\end{tabular}

epochs differ by 200 days. (Planetary and lunar positions were computed using analytic Poisson series. The details of how the orbital elements were derived can be found at http://www . projectpluto.com/jpl_eph.htm.) Then, the positions of asteroids were computed using orbital elements for the nearest epoch as in a two-body problem. This procedure is convenient as a first approximation. Since actual asteroids positions may differ from their computed ones far from the initial epoch due to several asteroid - asteroid encounters, we used only the same (short) period as in Paper I - from November 6, 1967 (JD 2439 800.5) to September 13, 2023 (JD 2460 200.5). In fact, some asteroids might have close encounters with any of the planets and thus their positions could be miscalculated using this simple approximation. However, this has a minor importance for our purposes due to the following three reasons:

1. Near terrestrial planets asteroids move very fast and approaches among asteroids occur at high velocities. Beyond the main belt, asteroids rarely encounter Jupiter. Just (5201) Ferraz-Mello and (6144) 1994 EQ3 were within a distance of $0.6 \mathrm{AU}$ from that planet.

2. Perturbations due to asteroids are negligible in comparison to those due to planets especially in the vicinity of the planet.

3. For the time just between the epochs - 100 days away - asteroid positions and their mutual distances were calculated twice - using both closest epochs. If the difference between mutual distances computed using different epochs exceeded $0.00005 \mathrm{AU}$ for a given asteroid pair and if this pair was about to reach its minimum separation distance $(r<0.005$ AU for asteroids with $H>9.5$ or $r<0.05$ AU for asteroids with at least one member brighter than absolute magnitude $H=9.5$ ) then orbital elements for a much closer epoch were used. There was practically no need to do that.

\section{Selection process}

The astrometric method of mass determination is done by measuring the deflection in position of the perturbed body from its ephemeris. The magnitude of the change in its mean motion $|\Delta n|$ depends on several quantities. Neglecting its orbital eccentricity and its mass we have

$|\Delta n| \simeq \frac{6 G m \epsilon}{a r v}$,

where $G$ is the universal constant of gravitation, $m$ is the mass of the perturber (depends on its diameter $D$ and bulk density $\rho$ ), $\epsilon$ is the energy efficiency of the close approach, the fraction of the change in the heliocentric velocity, which occurs in the tangential direction, $a$ is the semimajor axis of the perturbed asteroid, $r$ is the minimum separation distance between asteroids, and $v$ is their encounter velocity (see Carpino \& Knežević 1996). Three quantities, $D^{3}, r$ and $v$, may differ by several 
Table 3. Asteroids perturbed by (2) Pallas $(P \geq 6 \mathrm{~km} \mathrm{~s}, D=523 \mathrm{~km}$ ). Emphasized asteroids may probably be used for mass determination with current data.

\begin{tabular}{rlcccrc}
\hline \hline number & name & $\begin{array}{c}\text { date } \\
\text { year/m/d }\end{array}$ & $\begin{array}{c}r \\
{[\mathrm{AU}]}\end{array}$ & $\begin{array}{c}v \\
{\left[\mathrm{~km} \mathrm{~s}^{-1}\right]}\end{array}$ & $\begin{array}{c}P \\
{[\mathrm{~km} \mathrm{~s}]}\end{array}$ & $\begin{array}{c}\text { obs } \\
\text { year }\end{array}$ \\
\hline$(6995)$ & 1996 BZ1 & $1968 / 4 / 4$ & 0.009244 & 14.687 & 7.0 & 1978 \\
$\mathbf{( 2 2 0 4 )}$ & Lyyli & $1968 / 5 / 3$ & 0.023200 & 6.780 & 6.1 & 1943 \\
$(16972)$ & 1998 WK11 & $1968 / 5 / 9$ & 0.009665 & 14.352 & 6.9 & 1994 \\
$(21522)$ & 1998 MX11 & $1972 / 11 / 2$ & 0.013167 & 11.338 & 6.4 & 1991 \\
$\mathbf{( 7 6 7 1 )}$ & Albis & $1977 / 6 / 5$ & 0.003497 & 12.473 & 21.9 & 1969 \\
$(5470)$ & 1988 BK5 & $1979 / 12 / 13$ & 0.006511 & 12.837 & 11.4 & 1977 \\
$(\mathbf{2 0 9 2 9})$ & 2050 T-1 & $1984 / 11 / 10$ & 0.009646 & 7.405 & 13.4 & 1971 \\
$(\mathbf{3 1 3 1})$ & Mason-Dixon & $1984 / 12 / 4$ & 0.011895 & 10.845 & 7.4 & 1922 \\
$(20555)$ & 1999 RC115 & $1986 / 9 / 25$ & 0.004325 & 9.671 & 22.9 & 1990 \\
$(11161)$ & 1998 BA8 & $1991 / 4 / 23$ & 0.009354 & 12.804 & 8.0 & 1982 \\
$(23830)$ & 1998 QZ85 & $1993 / 10 / 27$ & 0.008444 & 16.832 & 6.7 & 1992 \\
$(18849)$ & 1999 RK55 & $1994 / 3 / 3$ & 0.007465 & 10.756 & 11.9 & 1987 \\
$(6752)$ & Ashley & $2000 / 6 / 25$ & 0.015028 & 8.093 & 7.9 & 1971 \\
$(21266)$ & 1996 HL25 & $2009 / 10 / 2$ & 0.009026 & 12.782 & 8.3 & 1996 \\
$(17278)$ & 2000 LK27 & $2014 / 4 / 14$ & 0.006341 & 14.418 & 10.5 & 1991 \\
$(3219)$ & Komaki & $2021 / 8 / 16$ & 0.011104 & 11.284 & 7.6 & 1934 \\
$(18544)$ & 1997 AA2 & $2021 / 11 / 1$ & 0.008950 & 10.474 & 10.2 & 1984 \\
\hline
\end{tabular}

orders of magnitude from one asteroid pair to the other. Thus, they play the major role in the $|\Delta n|$ determination.

We used the same method as in Paper I to find possible asteroid pairs that could be used for mass determinations. We searched for the largest values of the auxiliary $P$ parameter defined as

$P=\frac{D^{3}}{r v}$

where $D$ is in $\mathrm{km}, r$ is in $\mathrm{km}, v$ is in $\mathrm{km} \mathrm{s}^{-1}$, and $P$ is in $\mathrm{km} \mathrm{s}$. The method sensitivity to orbital eccentricity was not analysed.

Since $D$ was used mostly from Tedesco (1989) with several large asteroid diameters missing, other sources or guesses were also used occasionally, as in Paper I. For example, (64) Angelina is of type E, so its albedo is probably high; diameters for (88) Thisbe and (776) Berbericia were used from Bowell et al. (1994) containing the new IRAS catalog of asteroid diameters. In general, the new values for diameters are a bit smaller than those in Tedesco (1989) (e.g. diameter of (1) Ceres is about $848 \mathrm{~km}$ in comparison to $913 \mathrm{~km}$ ). But the results from other techniques, such as occultation of (2) Pallas, or HST images of (4) Vesta, indicate the new ones may be underestimated values. Moreover, the results of $P$ would not be lowered substantially even using the new diameters.

It must be noted that the $P$ parameter is not valid outside the sphere of action of the perturber. Its computation is just an approximation allowing comparisons between the encounters. For example, Hill's radius $R_{\mathrm{H}}$ is considered as

$R_{\mathrm{H}}=\sqrt[3]{\frac{m}{3 M}} \cdot r_{\mathrm{S}}$ where $m$ is the mass of the body, $M$ is the mass of Sun, and $r_{\mathrm{S}}$ is the distance from Sun (semimajor axis $a$ or perihelion distance $q$ ). $R_{\mathrm{H}}$ values are approximately $0.0100 \mathrm{AU}$ for Earth, 0.0072 AU for Mars, 0.3552 AU for Jupiter, but only 0.0015 AU for (1) Ceres, 0.0010 AU for (2) Pallas, 0.0009 AU for (4) Vesta.

\section{The big four main belt asteroids}

The mass of (1) Ceres has been determined by the astrometric method many times (e.g. Williams 1992; Carpino \& Knežević 1996; Michałak 2000). There is no need to list pairs with small $P$ here. The total number of those with $P \geq 6 \mathrm{~km} \mathrm{~s}$ is about 1000 in the period under study. It is only required that heavily perturbed asteroids due to (1) Ceres be listed and used to lower the uncertainty in its mass. Only some asteroids with $P \geq 60 \mathrm{~km} \mathrm{~s}$ are listed in Table 1 . All of these were observed quite well before and after the encounter and are therefore emphasized in Table 1. Similarly, observations of several other asteroids may improve our knowledge of the mass of (4) Vesta, which seems to be the second largest perturber in the main belt. In Table 2, we chose five heavily perturbed asteroids due to (4) Vesta $(P \geq 20 \mathrm{~km} \mathrm{~s})$ that were observed before and after the encounter. In fact, more than 300 pairs were found to have $P \geq 6 \mathrm{~km} \mathrm{~s}$.

As was mentioned in Paper I the fourth largest main belt asteroid (10) Hygiea may displace (2) Pallas in the total number of significant encounters. Indeed, this is confirmed if we compare the perturbations due to these two asteroids. While we found only 17 pairs with (2) Pallas (Table 3), there were 61 pairs with (10) Hygiea for $P \geq 6 \mathrm{~km} \mathrm{~s}$ (Tables 4 and 5). The summation of $P$ over all these encounters is $174.5 \mathrm{~km} \mathrm{~s}$ 
Table 4. Asteroids perturbed by (10) Hygiea before January 1, 2000. $P \geq 6 \mathrm{~km} \mathrm{~s}, D=429 \mathrm{~km}$. Emphasized asteroids may probably be used for mass determination with current data.

\begin{tabular}{|c|c|c|c|c|c|c|}
\hline number & name & $\begin{array}{c}\text { date } \\
\text { year/m/d }\end{array}$ & $\begin{array}{c}r \\
{[\mathrm{AU}]}\end{array}$ & $\begin{array}{c}v \\
{\left[\mathrm{~km} \mathrm{~s}^{-1}\right]}\end{array}$ & $\begin{array}{r}P \\
{[\mathrm{~km} \mathrm{~s}]}\end{array}$ & $\begin{array}{l}\text { obs } \\
\text { year }\end{array}$ \\
\hline (14375) & $1989 \mathrm{SU}$ & $1971 / 5 / 2$ & 0.027451 & 2.592 & 7.4 & 1976 \\
\hline (11 924) & 1992 WS3 & $1972 / 10 / 27$ & 0.022385 & 2.801 & 8.4 & 1990 \\
\hline (16036) & 1999 GV8 & $1972 / 12 / 8$ & 0.008438 & 4.939 & 12.7 & 1951 \\
\hline (9268) & 1978 VZ2 & $1972 / 12 / 15$ & 0.025907 & 2.474 & 8.2 & 1978 \\
\hline (16919) & 1998 FF35 & $1973 / 5 / 18$ & 0.008637 & 1.434 & 42.6 & 1990 \\
\hline (11 288) & $1990 \mathrm{XU}$ & $1973 / 5 / 31$ & 0.005207 & 5.764 & 17.6 & 1990 \\
\hline (4407) & Taihaku & $1978 / 1 / 13$ & 0.052457 & 1.522 & 6.6 & 1970 \\
\hline (16593) & 1992 UB3 & $1978 / 2 / 4$ & 0.012803 & 5.421 & 7.6 & 1955 \\
\hline (13 266) & 1998 QY30 & $1978 / 5 / 31$ & 0.014689 & 2.403 & 14.9 & 1992 \\
\hline (7362) & Rogerbyrd & $1978 / 6 / 11$ & 0.022725 & 3.634 & 6.4 & 1960 \\
\hline (10 818) & 1993 FK81 & $1979 / 5 / 29$ & 0.022368 & 3.798 & 6.2 & 1979 \\
\hline (22 108) & 2000 PD & $1982 / 3 / 25$ & 0.014267 & 3.757 & 9.9 & 1955 \\
\hline (3951) & Zichichi & $1983 / 8 / 16$ & 0.027718 & 2.765 & 6.9 & 1938 \\
\hline (21 248) & 1995 YP1 & $1983 / 9 / 23$ & 0.030090 & 1.154 & 15.2 & 1995 \\
\hline$(6143)$ & Pythagoras & $1983 / 12 / 19$ & 0.018749 & 2.464 & 11.4 & 1951 \\
\hline (1259) & Ógyalla & $1984 / 2 / 11$ & 0.034542 & 2.050 & 7.4 & 1928 \\
\hline (1780) & Kippes & $1984 / 5 / 14$ & 0.043080 & 1.901 & 6.4 & 1906 \\
\hline (20 638) & 1999 TV108 & $1988 / 7 / 26$ & 0.016438 & 1.714 & 18.7 & 1993 \\
\hline$(20371)$ & 1998 KE30 & $1988 / 11 / 8$ & 0.047163 & 1.467 & 7.6 & 1981 \\
\hline$(20540)$ & 1999 RV86 & $1989 / 3 / 3$ & 0.018664 & 2.723 & 10.4 & 1990 \\
\hline (12 141) & 4112 P-L & $1989 / 5 / 20$ & 0.041714 & 1.631 & 7.8 & 1960 \\
\hline (11 559) & 1993 FS23 & $1989 / 6 / 4$ & 0.020666 & 2.514 & 10.2 & 1990 \\
\hline (18215) & 4792 P-L & $1989 / 6 / 12$ & 0.033893 & 1.829 & 8.5 & 1960 \\
\hline (2619) & Skalnaté Pleso & $1989 / 12 / 11$ & 0.022379 & 1.707 & 13.8 & 1975 \\
\hline (12777) & 1994 QA1 & $1990 / 2 / 11$ & 0.013101 & 4.643 & 8.7 & 1990 \\
\hline (5457) & Queen's & $1993 / 6 / 10$ & 0.041282 & 1.864 & 6.9 & 1948 \\
\hline (22 880) & 1999 RL224 & $1994 / 9 / 3$ & 0.007061 & 3.701 & 20.2 & 1994 \\
\hline (17 109) & 1999 JF52 & $1994 / 10 / 31$ & 0.022559 & 3.619 & 6.5 & 1977 \\
\hline (6006) & Anaximandros & $1995 / 2 / 7$ & 0.009236 & 2.668 & 21.4 & 1972 \\
\hline (10788) & 1991 UC & $1995 / 5 / 3$ & 0.032804 & 2.419 & 6.7 & 1990 \\
\hline (11 215) & 1999 HN10 & $1995 / 5 / 31$ & 0.005374 & 3.441 & 28.5 & 1978 \\
\hline (465) & Alekto & $1995 / 12 / 25$ & 0.038037 & 1.509 & 9.2 & 1901 \\
\hline (15 487) & 1999 CC63 & $1996 / 5 / 25$ & 0.040184 & 1.297 & 10.1 & 1994 \\
\hline (3946) & Shor & $1998 / 3 / 30$ & 0.014400 & 0.917 & 40.0 & 1950 \\
\hline (2061) & Anza & $1999 / 4 / 4$ & 0.006464 & 9.419 & 8.7 & 1960 \\
\hline
\end{tabular}

for the former, and $754.6 \mathrm{~km} \mathrm{~s}$ for the latter. Thus, asteroid (10) Hygiea may be more than three times more effective on the motion of main belt asteroids (though comparison of the bulk densities may lower the difference). This is because the orbit of (2) Pallas is highly inclined to the ecliptic. As a result, asteroid encounters with (2) Pallas tend to occur at high speed and rarely result in significant perturbations. Emphasized asteroids in the tables are likely to be used for mass determination at present. Some of them have still not been tested by other authors. We used the combination of $P$, and time periods of astrometric observations pre- and post- encounter for a given pair, respectively, in order to denote asteroids suitable for mass determination. Neglecting the perturber's mass (here (2) Pallas and (10) Hygiea) emphasized asteroids may be deflected from their ephemerides by about $1^{\prime \prime}$ or more. We did not emphasize any asteroid without pre-encounter observations, nor asteroids that will be perturbed significantly in the future. The tables also contain unusual asteroids - (2204) Lyyli 
Table 5. Asteroids perturbed by (10) Hygiea after January 1, 2000. $P \geq 6 \mathrm{~km} \mathrm{~s}, D=429 \mathrm{~km}$. Most of them will probably be used for mass determination in the future.

\begin{tabular}{|c|c|c|c|c|c|c|}
\hline number & name & $\begin{array}{c}\text { date } \\
\text { year/m/d }\end{array}$ & $\begin{array}{c}r \\
{[\mathrm{AU}]}\end{array}$ & $\begin{array}{c}v \\
{\left[\mathrm{~km} \mathrm{~s}^{-1}\right]}\end{array}$ & $\begin{array}{r}P \\
{[\mathrm{~km} \mathrm{~s}]}\end{array}$ & $\begin{array}{l}\text { obs } \\
\text { year }\end{array}$ \\
\hline (1965) & van de Kamp & $2000 / 10 / 15$ & 0.021421 & 3.289 & 7.5 & 1927 \\
\hline (10380) & Berwald & $2001 / 1 / 5$ & 0.013185 & 3.364 & 11.9 & 1949 \\
\hline (5941) & Valencia & $2001 / 2 / 15$ & 0.017958 & 2.964 & 9.9 & 1972 \\
\hline (15 187) & $2112 \mathrm{~T}-2$ & $2003 / 12 / 17$ & 0.008236 & 4.096 & 15.6 & 1973 \\
\hline (17 207) & 2000 AW126 & $2004 / 5 / 22$ & 0.018852 & 3.549 & 7.9 & 1954 \\
\hline (24 433) & $2000 \mathrm{CF} 83$ & $2004 / 6 / 19$ & 0.003165 & 4.216 & 39.5 & 1988 \\
\hline (3030) & Vehrenberg & $2005 / 7 / 24$ & 0.021885 & 3.695 & 6.5 & 1962 \\
\hline (75) & Eurydike & $2005 / 9 / 16$ & 0.013484 & 5.647 & 6.9 & 1864 \\
\hline (11 054) & $1991 \mathrm{FA}$ & $2005 / 11 / 3$ & 0.014154 & 6.250 & 6.0 & 1937 \\
\hline (7487) & 1994 YM & $2006 / 6 / 8$ & 0.011351 & 6.883 & 6.8 & 1954 \\
\hline (15 567) & Giacomelli & $2006 / 6 / 14$ & 0.026796 & 1.915 & 10.3 & 1994 \\
\hline (5957) & Irina & $2007 / 1 / 3$ & 0.006555 & 7.622 & 10.6 & 1988 \\
\hline (6579) & 1981 ES4 & $2010 / 5 / 29$ & 0.014936 & 5.274 & 6.7 & 1953 \\
\hline (11 054) & $1991 \mathrm{FA}$ & $2011 / 5 / 23$ & 0.009320 & 6.301 & 9.0 & 1937 \\
\hline (14 094) & 1997 OJ1 & $2011 / 12 / 19$ & 0.005030 & 3.140 & 33.4 & 1996 \\
\hline (12936) & 2549 P-L & $2012 / 3 / 21$ & 0.032637 & 2.593 & 6.2 & 1960 \\
\hline (13 646) & $1996 \mathrm{HC} 12$ & $2012 / 3 / 23$ & 0.010860 & 2.138 & 22.7 & 1971 \\
\hline (10 018) & 1979 MG4 & $2015 / 5 / 24$ & 0.023737 & 1.425 & 15.6 & 1979 \\
\hline (20331) & 1998 HH45 & $2016 / 6 / 3$ & 0.041641 & 2.052 & 6.2 & 1997 \\
\hline (11 328) & 1995 UL & $2016 / 10 / 30$ & 0.023988 & 2.188 & 10.1 & 1986 \\
\hline (4803) & Birkle & $2017 / 4 / 5$ & 0.011922 & 2.069 & 21.4 & 1950 \\
\hline (22 769) & 1999 BD4 & $2019 / 10 / 28$ & 0.040339 & 1.315 & 9.9 & 1978 \\
\hline (16323) & $1107 \mathrm{~T}-2$ & $2020 / 12 / 23$ & 0.017165 & 2.594 & 11.9 & 1973 \\
\hline (1160) & Illyria & $2022 / 1 / 24$ & 0.016898 & 4.939 & 6.3 & 1929 \\
\hline (13634) & 1995 WY41 & $2022 / 6 / 10$ & 0.018540 & 2.944 & 9.7 & 1970 \\
\hline (12095) & $1998 \mathrm{HE} 102$ & $2022 / 12 / 11$ & 0.049546 & 1.672 & 6.4 & 1992 \\
\hline
\end{tabular}

encountered (2) Pallas, while (2061) Anza encountered (10) Hygiea. (11054) 1991 FA will have two encounters with (10) Hygiea.

\section{Other large perturbers}

The observed positions of some small numbered main belt asteroids may be deflected from their ephemerides even after perturbations due to the four largest main belt asteroids are included. This is because there are several other large perturbers in the belt, the masses of which are usually neglected. In general, we need precise long-term (pre- and post-encounter) astrometric observations of both encountered asteroids to reveal deflection and determine perturber's mass. However, special care must be taken not to neglect perturbations due to other sources during such extended periods of time.

Our search for large perturbers (other than the four largest ones) within the main belt was limited to bodies with absolute magnitude $H \leq 9.5$. We found a lot of pairs with $P \geq 5 \mathrm{kms}$. Those that occurred before 1980 are summarized in chronological order in Table 6, while those that occurred between 1980 and 1997 (including these years) are in Table 7. The rest (in 1998 and later) are listed at http://www. uniba.sk/ galad/massdet.htm. These could only be used for mass determination after several years. The policy of denoting asteroids in Table 6 is similar to the one used in the previous section except for the fact that we emphasized the perturbers instead of perturbed asteroids. In Table 7, we emphasized asteroids suitable for mass determination this decade (before 2010). All asteroids listed in the tables are from the main belt. Unfortunately, no pairs were found among the Trojans.

Altogether we emphasized 13 different asteroids (asteroid (16) Psyche is emphasized twice and (52) Europa three times) that could be tested for mass determination at present or by the end of this decade. This inference is based only on $P$ values and the date of the first astrometrical data available in the Minor Planet Center for both asteroids (last column in tables). However, some measurements are of low quality, unreliable, or poorly distributed before the encounter. In such cases, 
Table 6. Perturbers (first column) other than big four main belt asteroids and perturbed asteroids (third column) before January 1,1980 $(P \geq 5 \mathrm{~km} \mathrm{~s})$. Masses of emphasized asteroids may probably be determined with current data. $D$ is (assumed) perturber's effective diameter.

\begin{tabular}{|c|c|c|c|c|c|c|c|c|c|}
\hline & $N_{1}$ & $\begin{array}{c}D \\
{[\mathrm{~km}]}\end{array}$ & & $N_{2}$ & $\begin{array}{c}\text { date } \\
\text { year/m/d }\end{array}$ & $\begin{array}{c}r \\
{[\mathrm{AU}]}\end{array}$ & $\begin{array}{c}v \\
{\left[\mathrm{~km} \mathrm{~s}^{-1}\right]}\end{array}$ & $\begin{array}{r}P \\
{[\mathrm{~km} \mathrm{~s}]}\end{array}$ & $\begin{array}{l}\text { obs } \\
\text { year }\end{array}$ \\
\hline (29) & Amphitrite & 219 & (13 892) & $1266 \mathrm{~T}-2$ & $1967 / 11 / 24$ & 0.007938 & 1.578 & 5.6 & 1973 \\
\hline (704) & Interamnia & 333 & (14 100) & Weierstrass & $1968 / 7 / 2$ & 0.002303 & 6.456 & 16.6 & 1991 \\
\hline (39) & Laetitia & 159 & (2416) & Sharonov & $1968 / 1 / 3$ & 0.002459 & 1.976 & 5.5 & 1916 \\
\hline (11) & Parthenope & 162 & (17) & Thetis & $1968 / 2 / 18$ & 0.001796 & 2.297 & 6.9 & 1852 \\
\hline (511) & Davida & 337 & (7191) & 1993 MA1 & $1969 / 7 / 16$ & 0.004306 & 5.979 & 9.9 & 1949 \\
\hline (45) & Eugenia & 214 & (17 763) & $1998 \mathrm{EG}$ & $1969 / 7 / 23$ & 0.007365 & 0.577 & 15.4 & 1994 \\
\hline (24) & Themis & 205 & $(22491)$ & 1997 GX32 & $1969 / 9 / 9$ & 0.005852 & 1.395 & 7.1 & 1997 \\
\hline (45) & Eugenia & 214 & (15 167) & 2000 GS135 & $1970 / 1 / 21$ & 0.009186 & 0.895 & 8.0 & 1990 \\
\hline (3) & Juno & 244 & (6817) & Pest & $1970 / 4 / 19$ & 0.001563 & 5.441 & 11.4 & 1982 \\
\hline (409) & Aspasia & 168 & (9347) & 1991 RY21 & $1970 / 5 / 12$ & 0.000623 & 4.274 & 11.9 & 1979 \\
\hline (15) & Eunomia & 272 & (16 693) & 1994 YC2 & $1970 / 5 / 20$ & 0.017941 & 1.173 & 6.4 & 1955 \\
\hline (52) & Europa & 312 & (1023) & Thomana & $1971 / 5 / 31$ & 0.006537 & 3.763 & 8.3 & 1924 \\
\hline (3) & Juno & 244 & (16 670) & 1994 AS2 & $1971 / 12 / 7$ & 0.003455 & 4.688 & 6.0 & 1994 \\
\hline (173) & Ino & 159 & (23 094) & 1999 XF143 & $1973 / 5 / 5$ & 0.001138 & 2.658 & 8.9 & 1990 \\
\hline (16) & Psyche & 264 & (13 206) & 1997 GC22 & $1974 / 7 / 18$ & 0.003876 & 0.743 & 42.7 & 1960 \\
\hline (324) & Bamberga & 242 & (11 482) & 1988 BW & $1974 / 10 / 30$ & 0.001517 & 6.893 & 9.1 & 1988 \\
\hline (511) & Davida & 337 & (1960) & Guisan & $1975 / 2 / 19$ & 0.007197 & 6.713 & 5.3 & 1955 \\
\hline (15) & Eunomia & 272 & (23 389) & $1181 \mathrm{~T}-3$ & $1975 / 7 / 20$ & 0.005183 & 3.090 & 8.4 & 1977 \\
\hline (24) & Themis & 205 & (2296) & Kugultinov & $1975 / 12 / 23$ & 0.015699 & 0.438 & 8.4 & 1941 \\
\hline (13) & Egeria & 215 & $\left(\begin{array}{lll}2 & 175\end{array}\right)$ & 1996 XJ27 & $1976 / 6 / 7$ & 0.001442 & 7.023 & 6.6 & 1996 \\
\hline (15) & Eunomia & 272 & (19524) & Acaciacoleman & 1976/ 8/ 1 & 0.024084 & 0.715 & 7.8 & 1975 \\
\hline (52) & Europa & 312 & (13 180) & 1996 HV19 & $1977 / 3 / 6$ & 0.008204 & 3.441 & 7.2 & 1976 \\
\hline (76) & Freia & 190 & (10383) & 1996 SR7 & $1977 / 3 / 16$ & 0.000653 & 5.181 & 13.6 & 1994 \\
\hline (52) & Europa & 312 & (24 290) & 1999 XS190 & $1977 / 8 / 31$ & 0.006515 & 4.904 & 6.4 & 1980 \\
\hline (12) & Victoria & 117 & (24 367) & $2000 \mathrm{AC} 126$ & $1978 / 9 / 4$ & 0.000259 & 6.956 & 5.9 & 1986 \\
\hline (532) & Herculina & 231 & (18 014) & 1999 JC121 & $1979 / 2 / 25$ & 0.002822 & 5.173 & 5.6 & 1993 \\
\hline (356) & Liguria & 135 & (20 567) & 1999 RS129 & $1979 / 4 / 27$ & 0.000414 & 6.497 & 6.1 & 1977 \\
\hline (16) & Psyche & 264 & $(6852)$ & $1985 \mathrm{CN} 2$ & $1979 / 9 / 10$ & 0.006830 & 1.956 & 9.2 & 1952 \\
\hline (324) & Bamberga & 242 & $(16035)$ & 1999 FX32 & $1979 / 10 / 10$ & 0.001969 & 8.211 & 5.9 & 1986 \\
\hline
\end{tabular}

masses could not be determined and the total number of candidates will probably be less than 13 . In general, uncertainties in mass determination are high from single encounters, but they tend to be lower from multiple ones.

The limit for $P$ is lower than in Paper I, but it is not guaranteed that all such pairs are found here. Some changes in $P$ (in comparison to Paper I) are due to changes in $D$, which was previously computed from albedo; e.g. pair (65) Cybele (526) Jena had $P<6 \mathrm{~km} \mathrm{~s}$ and would be missing here using the same limit. Some pairs were not used for mass determination by other authors. They are usually concentrated on low numbered asteroid encounters that occured several decades ago. If some positions are found in old archived photographic plates for high numbered asteroids, they could then be used for mass determination. The results from Paper I were compared to that of Hilton et al. (1996). The mass of (45) Eugenia computed by the astrometrical method could be of special interest for comparison to the mass derived by other ground-based technique, as a satellite of this object has been discovered.

Most of the asteroids are continually observed near their oppositions. The last observation dates are, therefore, variable and not listed in tables.

\section{Small perturbers}

Masses of asteroids with $H>9.5$ are difficult to reveal by the astrometrical method, as they are smaller than $100 \mathrm{~km}$ in diameter (their albedos are probably higher than 0.03). Thus, only extremely close and slow encounters could cause large $P$ values. In the previous section, we found only one asteroid candidate for mass determination with $H<9.5$ that is smaller than $100 \mathrm{~km}$ in diameter - in 2014 (445) Edna will be encountered 
Table 7. Perturbers (first column) other than big four main belt asteroids and perturbed asteroids (third column) from 1980 to 1997 ( $P \geq 5 \mathrm{~km} \mathrm{~s}$ ). Masses of emphasized asteroids may probably be derived in this decade.

\begin{tabular}{|c|c|c|c|c|c|c|c|c|c|}
\hline & $N_{1}$ & $\begin{array}{c}D \\
{[\mathrm{~km}]}\end{array}$ & & $N_{2}$ & $\begin{array}{c}\text { date } \\
\text { year/m/d }\end{array}$ & $\begin{array}{c}r \\
{[\mathrm{AU}]}\end{array}$ & $\begin{array}{c}v \\
{\left[\mathrm{~km} \mathrm{~s}^{-1}\right]}\end{array}$ & $\begin{array}{r}P \\
{[\mathrm{~km} \mathrm{~s}]}\end{array}$ & $\begin{array}{l}\text { obs } \\
\text { year }\end{array}$ \\
\hline (15) & Eunomia & 272 & (19994) & 1990 TJ15 & $1980 / 4 / 18$ & 0.018923 & 1.352 & 5.3 & 1985 \\
\hline$(511)$ & Davida & 337 & (18 085) & $2000 \mathrm{JZ14}$ & $1980 / 9 / 8$ & 0.010761 & 4.594 & 5.2 & 1986 \\
\hline (128) & Nemesis & 194 & (22 347) & 1992 SE13 & 1980/10/18 & 0.004070 & 2.393 & 5.0 & 1981 \\
\hline (24) & Themis & 205 & (11 474) & $1982 \mathrm{SM} 2$ & $1980 / 11 / 13$ & 0.001083 & 3.393 & 15.7 & 1982 \\
\hline (24) & Themis & 205 & (8700) & 1993 JL1 & $1981 / 2 / 1$ & 0.008747 & 0.614 & 10.7 & 1975 \\
\hline (65) & Cybele & 245 & (22920) & 1999 TF94 & $1982 / 3 / 31$ & 0.007481 & 2.462 & 5.3 & 1989 \\
\hline (121) & Hermione & 217 & (5750) & Kandatai & $1982 / 6 / 9$ & 0.001114 & 4.545 & 13.5 & 1970 \\
\hline (145) & Adeona & 155 & (6173) & Jimwestphal & $1982 / 12 / 1$ & 0.003096 & 1.120 & 7.2 & 1977 \\
\hline (52) & Europa & 312 & (16 192) & 2000 AU207 & $1983 / 4 / 6$ & 0.007176 & 3.276 & 8.6 & 1978 \\
\hline (11) & Parthenope & 162 & (18 801) & 1999 JO76 & $1983 / 7 / 5$ & 0.001105 & 1.509 & 17.0 & 1992 \\
\hline (29) & Amphitrite & 219 & (17396) & 1981 EK45 & $1983 / 8 / 19$ & 0.004021 & 2.198 & 7.9 & 1981 \\
\hline (423) & Diotima & 217 & (12 146) & 6035 P-L & $1984 / 1 / 13$ & 0.002637 & 3.845 & 6.7 & 1960 \\
\hline (16) & Psyche & 264 & (9473) & Ghent & $1984 / 5 / 17$ & 0.009724 & 2.151 & 5.9 & 1993 \\
\hline (65) & Cybele & 245 & $(526)$ & Jena & $1984 / 6 / 24$ & 0.005867 & 3.330 & 5.0 & 1901 \\
\hline (16) & Psyche & 264 & (10908) & Kallestroetzel & $1985 / 4 / 21$ & 0.011500 & 1.862 & 5.7 & 1958 \\
\hline (29) & Amphitrite & 219 & (6904) & McGill & $1985 / 6 / 15$ & 0.009335 & 0.558 & 13.5 & 1990 \\
\hline (29) & Amphitrite & 219 & (14 809) & 1981 ES28 & $1986 / 1 / 17$ & 0.005391 & 2.467 & 5.3 & 1981 \\
\hline (451) & Patientia & 230 & (18715) & 1998 HE121 & $1986 / 2 / 20$ & 0.005351 & 1.254 & 12.1 & 1969 \\
\hline (15) & Eunomia & 272 & (20 784) & 2000 RN56 & $1986 / 7 / 7$ & 0.006294 & 3.081 & 6.9 & 1981 \\
\hline (704) & Interamnia & 333 & (21 868) & 1999 TK291 & 1987/ 1/11 & 0.008407 & 5.565 & 5.3 & 1997 \\
\hline (16) & Psyche & 264 & $(20874)$ & 2000 VL49 & $1987 / 3 / 20$ & 0.010296 & 2.064 & 5.8 & 1992 \\
\hline (52) & Europa & 312 & (4140) & Branham & $1987 / 10 / 8$ & 0.010997 & 3.295 & 5.6 & 1929 \\
\hline (9) & Metis & 184 & (7684) & Marioferrero & $1989 / 2 / 15$ & 0.002479 & 2.752 & 6.1 & 1983 \\
\hline (15) & Eunomia & 272 & (3591) & Vladimirskij & $1989 / 2 / 27$ & 0.003816 & 5.553 & 6.3 & 1932 \\
\hline (9) & Metis & 184 & (9362) & 1992 FE1 & $1989 / 7 / 26$ & 0.005891 & 1.174 & 6.0 & 1992 \\
\hline (45) & Eugenia & 214 & (673) & Edda & $1990 / 8 / 13$ & 0.003766 & 2.548 & 6.8 & 1908 \\
\hline (16) & Psyche & 264 & $(19462)$ & Ulissedini & $1991 / 8 / 19$ & 0.029378 & 0.762 & 5.5 & 1980 \\
\hline$(511)$ & Davida & 337 & (11985) & $1995 \mathrm{VG}$ & $1991 / 10 / 14$ & 0.004396 & 6.155 & 9.5 & 1981 \\
\hline (7348) & 1993 FJ22 & 18.2 & (7562) & Kagiroino-Oka & $1993 / 3 / 26$ & 0.000001 & 1.673 & $24.2^{a}$ & 1986 \\
\hline (15) & Eunomia & 272 & (10324) & Vladimirov & $1993 / 7 / 10$ & 0.006067 & 3.264 & 6.8 & 1990 \\
\hline (52) & Europa & 312 & (124) & Alkeste & 1993/10/17 & 0.012436 & 2.585 & 6.3 & 1872 \\
\hline (52) & Europa & 312 & $(13$ 151) & Polino & $1993 / 10 / 28$ & 0.002112 & 4.250 & 22.6 & 1995 \\
\hline (19) & Fortuna & 166 & $(18$ 162) & 2000 PX15 & 1993/12/ 1 & 0.001684 & 3.608 & 5.0 & 1993 \\
\hline (29) & Amphitrite & 219 & (987) & Wallia & $1994 / 3 / 3$ & 0.002452 & 3.198 & 9.0 & 1899 \\
\hline (56) & Melete & 117 & $(22960)$ & 1999 UE4 & $1994 / 9 / 23$ & 0.000344 & 4.402 & 7.1 & 1994 \\
\hline (19) & Fortuna & 166 & (3486) & Fulchignoni & $1996 / 5 / 14$ & 0.002135 & 2.304 & 6.2 & 1952 \\
\hline (704) & Interamnia & 333 & $(7461)$ & Kachmokiam & $1997 / 5 / 31$ & 0.007478 & 5.285 & 6.2 & 1984 \\
\hline (13) & Egeria & 215 & (14 689) & 2000 AM2 & $1997 / 7 / 21$ & 0.000801 & 4.015 & 20.7 & 1973 \\
\hline
\end{tabular}

${ }^{a}$ Probably an upper limit. A very uncertain value due to $r<<$ and assumed albedo of 0.04 .

by (1764) Cogshal at a minimum distance of just $0.000052 \mathrm{AU}$. In spite of the fact that we know little about effective diameters of small perturbers with $H>9.5$, it seems that only one pair was found with $P \geq 5 \mathrm{~km} \mathrm{~s}$. This is the pair (7348) $1993 \mathrm{FJ} 22-$ (7562) Kagiroino-Oka, which had the closest encounter of all computed pairs. In 1993 they were only about $200 \mathrm{~km}$ apart! (It should be noted that the uncertainties in the ephemerides for the objects are probably several times greater than this.) The pair is included in Table 7. However, the sparse astrometric data for (7562) Kagiroino-Oka prior to the encounter, 
combined with the extreme uncertainty in the minimum separation, render this encounter unsuitable for mass computation.

\section{Conclusions}

We tried to find asteroids for which masses could be determined by the astrometric method. We concentrated on those small asteroids that could manifest quick ephemeris deviations after a close encounter to a larger asteroid. The masses of the largest perturbers among main belt asteroids - (1) Ceres and (4) Vesta - could be determined more reliably from several heavily perturbed bodies. In the case of the other two large perturbers we conclude that perturbations due to (10) Hygiea are more effective on the motion of main belt asteroids than those due to (2) Pallas by a factor of three or more. At this time, however, the latter is used in asteroid orbit determinations along with the planets, Moon, and two large asteroids (1) Ceres and (4) Vesta while the former is normally not (except for special cases). We found also several small asteroids that could be used for reliable mass determination of (10) Hygiea.

In addition to the four largest main belt asteroids, there are others - (11) Parthenope, (13) Egeria, (15) Eunomia, (16) Psyche, (24) Themis, (29) Amphitrite, (39) Laetitia, (45) Eugenia, (52) Europa, (65) Cybele, (121) Hermione, (451) Patientia, (511) Davida - the masses of which could probably be achieved by the end of this decade using astrometric data of smaller asteroids. However, if the astrometric measurements of the small asteroids before the important encounter with the perturber are unreliable, of low quality, or poorly distributed, it would result in reducing the number of perturbers listed above. In general, high numbered asteroids will be used more thoroughly for mass determination of quite large asteroids over the next decade. However, in some cases, useful data for them may be found in old photographic plates.

Acknowledgements. This work was supported by Grant VEGA 7157 and Comenius University Grant (No. UK/100/2001).

\section{References}

Bowell, E., Muinonen, K., \& Wasserman, L. H. 1994, in Asteroids, Comets, Meteors 1993, ed. A. Milani, M. Di Martino, \& A. Celino (Kluwer Academic Publishers, Belgirate), 477

Brown, M. E., \& Margot, J. L. 2001, IAUC 7588

Carpino, M., \& Knežević, Z. 1996, in Proc. of the 1st Italian Meet. of Planetary Science, Bormio, Italy

Galád, A. 2001, A\&A, 370, 311

Hilton, J. L., Seidelmann, P. K., \& Middour, J. 1996, AJ, 112(5), 2319

Merline, W. J., Close, L. M., Dumas, C., et al. 1999, IAUC, 7129

Merline, W. J., Close, L. M., Shelton, J. C., et al. 2000, IAUC, 7503

Merline, W. J., Menard, F., Close, L., et al. 2001, IAUC, 7703

Michałak, G. 2000, A\&A, 360, 363

Michałak, G. 2001, A\&A, 374, 703

Storrs, A., Vilas, F., Landis, R., et al. 2001, IAUC, 7599

Tedesco, E. F. 1989, in Asteroids II, ed. R. P. Binzel, T. Gehrels, \& M. S. Matthews (The Univ. of Arizona Press, Tucson), 1090

Thomas, P. C., Veverka, J., Bell, J. F., et al. 1999, Icarus, 140, 17

Viateau, B., \& Rapaport, M. 2001, A\&A, 370, 602

Williams, G. V. 1992, in Asteroids, Comets, Meteors 1991, ed. A. W. Harris, \& E. Bowell (Lunar and Planetary Institute, Houston), 641 